\title{
Relevamiento de la concentración de arsénico total en agua proveniente de varias fuentes en una zona arrocera del Uruguay
}

Survey of the total arsenic concentration in the water coming from several sources in a rice area of Uruguay

RECIBIDO: $17 /$ 10/2018 $\rightarrow$ APROBADO: 27/11/2018 Mlfalchi@fq.edu.uy

i̊ FAlchi, lucía (1); Pizzorno, PAUlina (2); iaquinta, Fiorella (1); COUSILlas, ADRIANA (2)

(1) ÁREA químicA ANALítica, DePARTAMENTo ESTRElla CAMPos, FACULTAD DE QUÍMICA, UNIVERSIDAD DE LA REPÚBLICA, MONTEVIDEO, URUGUAY.

(2) Cequimtox. ÁREA TOXiCOlogía, DePARTAMENTo ESTRElla CAmpos, FACULTAD DE QUímiCA, UNIVERSIDAd DE LA REPÚBLICA, MONTEVIDEO, URUGUAY.

\section{RESUMEN}

El Arsénico (As) es un elemento natural de la corteza terrestre de gran relevancia toxicológica debido a los efectos nocivos sobre la salud que provoca su exposición. Los elevados niveles de As en agua subterránea en Argentina y Chile son ampliamente estudiados y conocidos, no obstante, en Uruguay existen escasos estudios de investigación sobre el tema. En este trabajo se realizó la determinación de As total en muestras de agua mediante espectrometría de absorción atómica con generación de hidruros (HG-AAS). La metodología se basó en el método normalizado de la American Public Health Association (APHA 3114 B) y se evaluó la linealidad, la precisión y la veracidad como parámetros de desempeño para su implementación. Fueron analizadas 49 muestras de agua superficial y subterránea provenientes de una zona del país que se caracteriza por el cultivo de arroz. Los resultados obtenidos no superan el nivel de As establecido por la normativa uruguaya $\left(20 \mu \mathrm{g} \mathrm{\textrm {L } ^ { - 1 } )}\right.$ y se observan valores más elevados en aguas subterráneas que en las superficiales. Este trabajo es un primer aporte para conocer los niveles de As total en agua que se utiliza para uso doméstico y en los cultivos de arroz.

PALABRAS CLAVE: As, agua subterránea, arroz, HG-AAS.

\section{ABSTRACT}

Arsenic (As) is a natural element of the earth's crust. Due to the health effects caused by its exposure, it has a great relevance in toxicological studies. The high 
levels of As in groundwater in Argentina and Chile are widely studied and known. However, in Uruguay there are few research studies based on the levels of As in water. In this work the determination of the total As in water samples was carried out, using atomic absorption spectrometry with hydride generation (HGAAS). The methodology was based on the standardized method of the American Public Health Association APHA 3114 B. We evaluated linearity, accuracy and veracity as performance parameters for its implementation. A total of 49 samples of surface and underground water were analyzed. The results did not exceed the standards level established in Uruguayan regulations (20 $\mu \mathrm{g} \mathrm{L-1).} \mathrm{Also,} \mathrm{higher}$ values were observed in groundwater rather than in surface water. This research is an initial work to know the As levels in water used for domestic purposes and rice plantations in Uruguay.

KEYWORDS: As, underground water, rice, HG-AAS.

\section{INTRODUCCIÓN}

El Arsénico (As) es un metaloide presente en la naturaleza, ampliamente distribuido en la corteza terrestre: en agua, aire y suelo. Es capaz de formar compuestos orgánicos e inorgánicos y presentarse en distintos estados de oxidación $(-\mathrm{III}, 0,+\mathrm{III},+\mathrm{V})$. Las especies químicas más relevantes del As son: arseniato $[\mathrm{As}(\mathrm{V})]$, arsenito $[\mathrm{As}(\mathrm{III})]$, formas metiladas de As V y As III, arsenobetaína, arsenocolina, arsenoazúcares y arsenolípidos. Las especies inorgánicas de As son las de mayor toxicidad frente a las orgánicas (WHO, 2001; WHO, 2011; Litter, et al., 2009). La Agencia para las sustancias tóxicas y el registro de enfermedades (Agency for Toxic Substances and Disease Registry, ATSDR) ubica al As en el primer lugar de la lista de prioridad de sustancias toxicológicamente relevantes (ATSDR, 2017). La Organización Mundial de la Salud (OMS) lo considera dentro de las diez sustancias más preocupantes para la salud pública (WHO, 2018) y el Centro Internacional de Investigaciones sobre Cáncer (International Agency for Research on Cancer, IARC) ha clasificado al As como cancerígeno para seres humanos (IARC, 2014).

Las principales vías de exposición son el aire, el agua, el suelo y los alimentos. A excepción de la contaminación antropogénica, los mayores niveles de As se han encontrado en los recursos hídricos subterráneos, debido a los procesos de interacción natural de agua-roca y a la elevada relación de sólidos en solución que se encuentra en los acuíferos (Mañay, 2013).

El principal problema para la salud pública radica en el uso de agua contaminada, para beber, preparar alimentos y regar cultivos agropecuarios. Los síntomas de intoxicación aguda involucran principalmente entumecimiento de los pies y las manos, calambres musculares y ocasionalmente la muerte. La exposición crónica a bajos niveles de As inorgánico debido a la ingesta de agua contaminada incluye: cambio de pigmentación de la piel, lesiones cutáneas, hiperqueratosis, además de cáncer de piel, vejiga, pulmón y riñones (WHO, 2018; Mañay, 2013; Spratlen, 2017). 
La presencia de altos niveles de As en el agua es un tema prioritario de preocupación ambiental, ya que limita el uso del recurso de agua potable e impide el crecimiento socioeconómico, la sostenibilidad del uso racional de los suelos y el desarrollo sostenible de la agricultura (Litter, et al., 2009). Esta preocupación, junto con los efectos tóxicos en la salud humana y animal y las consecuentes repercusiones patológicas asociadas con su exposición, ha llevado a profundizar su estudio y desarrollar diversas metodologías analíticas capaces de estudiar al As en sus diferentes formas y concentraciones, incluso a niveles de traza. Existen diversas metodologías para la determinación de As; la espectrometría de absorción atómica por generación de hidruros (HG-AAS) y la espectrometría de masas con plasma acoplado inductivamente (ICP-MS) son las más utilizadas hoy en día, ya que son compatibles para la detección y cuantificación de As de acuerdo con los límites impuestos por la legislación para su contenido en agua potable. En la mayoría de los países este límite se ha fijado recientemente en $10 \mu \mathrm{g} \mathrm{L}^{-1}$ como valor objetivo de acuerdo a las recomendaciones de la OMS (WHO, 2018; Litter, et al., 2009).

Debido a la introducción de estos nuevos límites internacionales, la determinación de As en agua comenzó a tener relevancia geoquímica-ambiental en Uruguay. En noviembre de 2011 se aprobó en el país el Decreto № 375/011 (Uruguay, 2011) que establece que la norma UNIT 833 (Instituto Uruguayo de Normas Técnicas, 2008) define los parámetros requeridos para el agua. Allí se determina que los niveles de As total deberán ser menores a $20 \mu \mathrm{g} \mathrm{L} \mathrm{L}^{-1}$, con el objetivo de alcanzar las recomendaciones de la OMS (Instituto Uruguayo de Normas Técnicas, 2008).

Dada la relevancia en la salud pública y el impacto ambiental que representa la presencia de As en agua en altas concentraciones, así como las escasas referencias bibliográficas sobre la situación en Uruguay, este estudio surge con el fin de realizar un relevamiento de sus niveles en una zona del país dedicada al cultivo de arroz altamente demandante de agua.

\section{MATERIALES Y MÉTODOS}

\section{Muestreo}

La región de muestreo se ubica al noreste de Uruguay, en el departamento de Treinta y Tres, y es reconocida por el cultivo de arroz. Se tomaron muestras dentro del predio de una empresa arrocera interesada en conocer los niveles de As del lugar. Los puntos de muestreo fueron aguas superficiales de la laguna Merín, de donde surgen los canales que abastecen a las plantaciones, y varios puntos en los canales. Además, se tomaron muestras de aguas subterráneas de los pozos que la suministran al poblado vecino y a las oficinas e instalaciones de la empresa arrocera. Se recolectaron muestras de agua en las localidades de Vergara y Cebollatí y en el río Cebollatí. Estas muestras de zonas aledañas se consideran control, ya que son localidades cercanas a la zona de interés y el agua proviene de la misma cuenca principal, la Laguna Merín o acuífero Chuy. Se realizó el muestreo de 
forma no probabilística por conveniencia, abarcando todas las áreas a las que se tuvo acceso dentro de la empresa arrocera y en las ciudades aledañas. En total se recolectaron 49 muestras.

Tanto la toma como la conservación de las muestras se realizó según recomendaciones de APHA (American Public Health Association, American Water Works Association y Water Environment Federation, 2012). Para la recolección de agua subterránea se limpió la tubería de salida dejándola correr unos 3 minutos y se hizo la toma de muestra. Las muestras de agua superficial fueron extraídas de preferencia en la zona central y de una zona donde fluía el agua pero sin turbulencia. Se evitó tomar agua de las márgenes del río, ya que allí no está perfectamente mezclada y puede haber sufrido efectos de evaporación o de contaminación. Las muestras fueron colectadas en frascos de polipropileno de $500 \mathrm{~mL}$ con la adición de ácido nítrico al $5 \% \mathrm{v} / \mathrm{v}$ para su conservación, transporte y posterior análisis en el laboratorio.

\section{Análisis}

La determinación de As total en las muestras de agua se realizó en el CEQUIMTOX (Centro Especializado en Química Toxicológica - Facultad de Química) mediante espectrometría de absorción atómica por generación de hidruros (HG-AAS), basada en el método normalizado de APHA 3114 B (American Public Health Association, American Water Works Association and Water Environment Federation, 2012). Se analizó cada muestra por duplicado, con un tratamiento inicial de digestión abierta utilizando ácido clorhídrico concentrado y posterior agregado del agente reductor ioduro de potasio $20 \% \mathrm{~m} / \mathrm{v}$.

Se verificó la metodología evaluando los siguientes parámetros de desempeño: linealidad, precisión, veracidad (muestras fortificadas a dos niveles y material de referencia certificado: LATU MRC. INO. 101), límites de detección y cuantificación.

\section{RESULTADOS Y DISCUSIÓN}

La metodología para la determinación de As se adaptó basándose en las recomendaciones de la APHA con el objetivo de trabajar dentro de los lineamientos de la química verde, con el uso de la menor cantidad de reactivos, con la menor concentración posible y generando la menor cantidad de residuos, en el menor tiempo de análisis. Se realizó una digestión en condiciones fuertemente ácidas para eliminar materia orgánica presente. Luego de la digestión las muestras se redujeron con el agregado de ioduro de potasio para obtener la forma adecuada de As para la generación de hidruros. Se estudiaron parámetros de desempeño para evaluar las modificaciones realizadas y los resultados obtenidos se incluyen en la Tabla 1. 
TABLA 1. Parámetros de desempeño.

\begin{tabular}{|cc|}
\hline Parámetros de desempeño & Resultado \\
\hline Linealidad $(0-12 \mu \mathrm{g} \mathrm{L}-1)$ & $\mathrm{R}^{2}>0.995$ \\
\hline Precisión $(\mathrm{n}=10, \mathrm{RSD})$ & $<10 \%$ \\
\hline Veracidad $(\mathrm{n}=10)$ & $75-125 \%$ \\
\hline LOD $(3 \mathrm{~s} / \mathrm{b}, \mathrm{n}=10)$ & $0,5 \mu \mathrm{g} \mathrm{L} \mathrm{L}^{-1}$ \\
\hline LOQ $(10 \mathrm{~s} / \mathrm{b}, \mathrm{n}=10)$ & $1,5 \mu \mathrm{g} \mathrm{L}-1$ \\
\hline
\end{tabular}

Los resultados de As total obtenidos por el análisis de las muestras de agua recogidas en el predio de la empresa se agrupan en la Tabla 2. Se clasifican según su origen, superficial o subterránea, y se indica la cantidad de muestras por punto de muestreo analizadas. Se presentan en ambos casos las concentraciones máximas y mínimas encontradas para cada grupo de muestras. A su vez, se indican en la Tabla 3 los resultados de As obtenidos en las muestras provenientes de la zona aledaña a la empresa arrocera. Allí se especifican las ciudades muestreadas, la cantidad de muestras obtenidas en cada una y los valores mínimos y máximos en cada grupo de muestras.

TABLA 2. Resultados de As en aguas provenientes del predio de la empresa arrocera.

\begin{tabular}{|cccccccc}
\hline \multicolumn{7}{|c|}{ Concentración promedio de As $\left(\mathbf{\mu g} \mathbf{L}^{-1}\right)$} \\
\hline \multicolumn{7}{|c|}{ Agua superficial } \\
\hline $\begin{array}{c}\text { Lagunas } \\
(\mathrm{n}=6)\end{array}$ & $\begin{array}{c}\text { Canales } \\
(\mathrm{n}=9)\end{array}$ & $\begin{array}{c}\text { Chacra } \\
(\mathrm{n}=7)\end{array}$ & $\begin{array}{c}\text { Pozo } \\
\text { ESCUELA } \\
(\mathrm{n}=2)\end{array}$ & $\begin{array}{c}\text { POZO } 1 \\
(\mathrm{n}=3)\end{array}$ & $\begin{array}{c}\text { POZO } 2 \\
(\mathrm{n}=9)\end{array}$ & $\begin{array}{c}\text { POZO } 3 \\
(\mathrm{n}=4)\end{array}$ & $\begin{array}{c}\text { POZO } 4 \\
(\mathrm{n}=2)\end{array}$ \\
\hline$<1,5$ & $<1,5$ & $<1,5$ & 5,6 & 9,5 & 6,3 & 7,9 & 2,2 \\
\hline $\begin{array}{c}\text { Mín/Máx } \\
<0,5 / 2,2\end{array}$ & $\begin{array}{c}\text { Mín/Máx } \\
<0,5 / 2,4\end{array}$ & $\begin{array}{c}\text { Mín/Máx } \\
<0,5 / 2,4\end{array}$ & $\begin{array}{c}\text { Mín/Máx } \\
4,7 / 6,4\end{array}$ & $\begin{array}{c}\text { Mín/Máx } \\
8,9 / 10,3\end{array}$ & $\begin{array}{c}\text { Mín/Máx } \\
5,4 / 8,2\end{array}$ & $\begin{array}{c}\text { Mín/Máx } \\
6,3 / 10,5\end{array}$ & $\begin{array}{c}\text { Mín/Máx } \\
1,2 / 2,8\end{array}$ \\
\hline
\end{tabular}

TABLA 3. Resultados de As en las zonas aledañas.

\begin{tabular}{|c|c|c|c|}
\hline \multicolumn{4}{|c|}{ Concentración promedio de As $\left(\mu \mathrm{g} \mathrm{L}^{-1}\right)$ en zonas aledañas } \\
\hline Vergara & \multicolumn{2}{|c|}{ Cebollatí } & Río Cebollatí \\
\hline \multirow{2}{*}{$\begin{array}{c}\text { Ciudad } \\
(\mathrm{n}=1)<0,5\end{array}$} & Ciudad: punto $1(\mathrm{n}=2)$ & Ciudad: punto $2(\mathrm{n}=3)$ & $(\mathrm{n}=1)$ \\
\hline & 4,9 & 10,7 & 6,5 \\
\hline --Mín/Máx & \multicolumn{2}{|c|}{ Mín/Máx } & Mín/Máx \\
\hline
\end{tabular}


La norma nacional UNIT 833 (Instituto Uruguayo de Normas Técnicas, 2008) determina el valor máximo permitido de As en $20 \mu \mathrm{g} \mathrm{L} \mathrm{L}^{-1}$ para agua potable. Como se observa en los resultados tanto de las aguas superficiales como de las subterráneas, las concentraciones promedio de As obtenidas cumplen con la especificación. Esto es de significativa relevancia, ya que las aguas subterráneas de pozo son las que abastecen a la población de la zona y no se observa anomalía en la concentración de As que consumen.

Al comparar los resultados entre las aguas superficiales y subterráneas se observa diferencia entre las concentraciones encontradas en ellas. Las muestras provenientes de aguas superficiales en su mayoría poseen concentraciones bajas, menores a

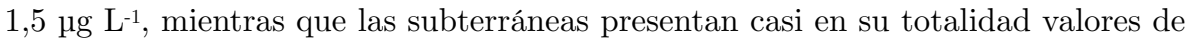

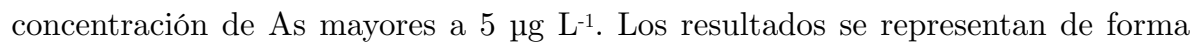
gráfica en la Figura 1, donde se dividió el rango de concentraciones de As obtenidas según las normativas legales y se evalúa si el mayor aporte de As proviene de aguas superficiales o subterráneas. Se observa claramente que para concentraciones desde el nivel de cuantificación hasta $20 \mu \mathrm{g} \mathrm{L}{ }^{-1}$ el aporte que prima siempre es de origen subterráneo. Esta observación se puede deber a procesos de origen geogénico, a la composición y condiciones de suelo subterráneo. En general, el pH del suelo es levemente alcalino y junto con las condiciones reductoras se favorece la desorción del As desde fases adsorbidas del suelo, vertiéndose en el agua presente (Smedley y Kinniburgh, 2002).

La concentración de As encontrada tanto para aguas superficiales y subterráneas en el predio de la empresa arrocera, en el pueblo vecino y las zonas aledañas es en general similar, por lo que se podría atribuir la presencia de As en la zona a su proveniencia natural desde los suelos de la zona.

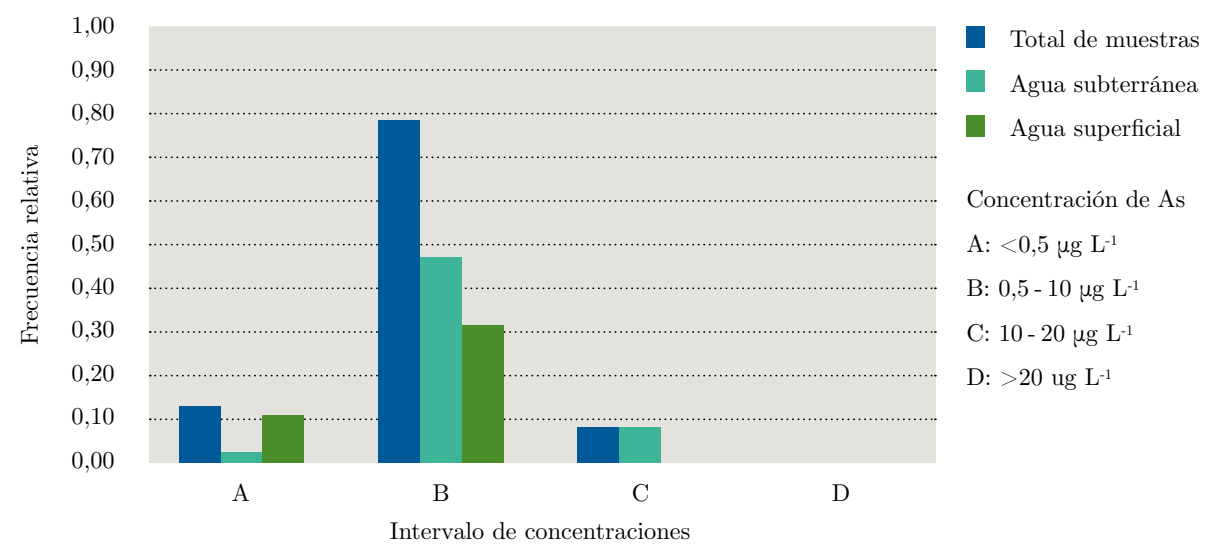

Figura 1. Frecuencia relativa de aguas superficiales y subterráneas frente al total de muestras dependiendo de la concentración de As total.

Según la OMS, el valor máximo permitido para As total es $10 \mu \mathrm{g} \mathrm{L} \mathrm{L}^{-1}$. Este valor es el objetivo planteado según la norma nacional a implementarse como límite en un futuro cercano. Llas concentraciones de As de algunas muestras analizadas en este trabajo, provenientes de pozos subterráneos que abastecen a la población, sobrepasan 
ese valor. En Uruguay más de un tercio de la población utiliza agua subterránea para consumo doméstico (OSE, s.d.) y es un recurso ampliamente utilizado en el sector agroproductivo, por lo que el estudio de la calidad de agua en cuanto a su contenido de As es de gran relevancia.

Existen escasos estudios de investigación hoy en día en Uruguay. Son conocidos los relevamientos de Manganelli (2006) o Mañay (2013), por ejemplo, pero se encuentran en general relacionados a zonas del sur o suroeste de Uruguay. Por ello, este trabajo es un aporte inicial para conocer los niveles de As en agua de consumo humano en una zona puntual del noroeste del país no estudiada anteriormente, dando paso a que se inicien nuevos proyectos de investigación para su estudio en todo Uruguay.

Dada la conocida toxicidad de la especie inorgánica de As III y su estrecha relación con patologías en seres humanos, se continuará este trabajo con el desarrollo de la metodología para la determinación de las especies de As en agua. Luego se estudiará el grado de toxicidad y se realizará una evaluación de riesgo poblacional con el fin de aportar información a la salud pública.

\section{CONCLUSIONES}

Este estudio es un primer aporte al conocimiento de los niveles de As total en agua que se utiliza para consumo poblacional y de riego en plantaciones de arroz en una zona puntual del noreste de Uruguay. Si bien las concentraciones de As total se encontraron dentro de especificaciones de normativas nacionales, se encontraron concentraciones de As que sobrepasan los niveles objetivos del país para aguas potables de consumo humano.

\section{REFERENCIAS}

American Public Health Association, American Water Works Association y Water Environment Federation, 2012. Standard methods for the examination of water and wastewater. 22va ed. Washington: APHA. Standard Method 3114 CL, Approved 2009- Editorial Rev. 2011.

ATSDR, 2017. CERCLA Priority list of hazardous substances [En línea]. Atlanta: ATSDR. [Consulta: 12 de octubre, 2018]. Disponible en: https://www.atsdr.cdc. gov/spl/previous/07list.html

IARC, 2014. World cancer report. Lyon: IARC.

Instituto Uruguayo de Normas Técnicas, 2008. UNIT 833: Agua potable-Requisitos. Montevideo: UNIT.

Litter, M., Armienta, M.A. y Farias, S.S., eds., 2009. IBEROARSEN. Metodologías analíticas para determinación y especiación de As en aguas y suelos. Buenos Aires: CYTED.

Manganelli, A., Goso, C., Guerequiz, R., Fernández-Turiel, J.L., García Vallès, M. y Gimeno, D., 2006. Estudio preliminar del contenido de arsénico de las aguas subterráneas del suroeste de Uruguay. En: Geogaceta, 41, pp.115-118. 
Mañay, N., Goso, C., Pistón, M., Fernández-Turiel, J.L., García-Vallés, M., Rejas, M. y Guerequiz, R., 2013. Groundwater arsenic content in Raigón aquifer system (San José, Uruguay). En: Revista SUG, 38, pp.20-38.

OSE, [s.d.]. Agua subterránea [En línea]. Montevideo: OSE. [Consulta: 12 de octubre de 2018]. Disponible en: http://www.ose.com.uy/agua/agua-subterranea

Smedley, P. y Kinniburgh, D., 2002. A review of the source, behaviour and distribution of arsenic in natural waters. En: Applied Geochemistry, 17(5), pp.517-568.

Spratlen M.J., Gamble M.V., Grau-Pérez, M., Kuo, C.C., Best, L.G., Yracheta, J., Francesconi, K., Goessler, W., Mossavar-Rahmani, Y., Hall, M., Umans, J.G., Fretts, A., Navas-Acien, A. Arsenic metabolism and one-carbon metabolism at low-moderate arsenic exposure: Evidence from the strong heart study. En: Food and Chemical Toxicology Journal, 105, pp.387-397.

Uruguay. Decreto 129/009, de 03 de noviembre de 2011. Diario Oficial, 14 de setiembre de 2011, No. 28.348, p.11.

WHO, 2018. Arsenic [En línea]. Geneva: WHO. [Consulta: 12 de octubre de 2018]. Disponible en: http://www.who.int/mediacentre/factsheets/fs372/en/

WHO, 2011. Guidelines for drinking-water quality [En línea]. Geneva: WHO.

[Consulta: 12 de octubre de 2018]. Disponible en: http://www.who.int/water sanitation_health/publications/2011/9789241548151_toc.pdf

WHO, 2001. Arsenic and arsenic compounds [En línea]. Geneva: WHO.

(Enviromental Health Criteria, 224). [Consulta: 12 de octubre de 2018]. Disponible en: http://apps.who.int/iris/bitstream/handle/10665/42366/WHO EHC_224.pdf;jsessionid=1FC9D7D022CC57D77AAD352F36A11B55? sequence =1 